\title{
Correlation of measured neon soft X-ray pulses of the INTI plasma focus with the reflected shock phase at $12 \mathrm{KV}$
}

\author{
Federico A. Roy Jr ${ }^{*}$ \\ Faculty of Science Information Technology and Mathematics (FOSTEM) \\ Centre for Plasma Research, Inti International University \\ Persiaran Perdana BBN, Putra Nilai Negeri Sembilan, Malaysia \\ federico.royjr@newinti.edu.my \\ Perk Lin Chong \\ Faculty of Science Information Technology and Mathematics (FOSTEM) \\ Centre for Plasma Research, Inti International University \\ Persiaran Perdana BBN, Putra Nilai Negeri Sembilan, Malaysia \\ chong.perklin@newinti.edu.my
}

Sor Heoh Saw

Centre for Plasma Research

Inti International University Persiaran Perdana BBN, Putra Nilai Negeri Sembilan, Malaysia Institute of Plasma Focus Studies, 32 Oakpark Drive, Chadstone, VIC3148, Australia sorheoh.saw@newinti.edu.my

Published 13 August 2014

\begin{abstract}
The six-phase Lee Model Code is used to fit the computed current waveform to the measured waveform of the INTI Plasma Focus (PF;2.2 kJ at $12 \mathrm{kV}$ ), a T2 PF device, operated as a source of Neon soft X-ray (SXR) with optimum yield around 2.5 - 3 Torr of neon. The characteristic He-like and H-like neon line SXR pulse is measured using a pair of SXR detectors with selected filters that, by subtraction, have a photon energy window of 900 to $1550 \mathrm{eV}$ covering the region of the characteristic neon SXR lines. The aim of this paper is to investigate the correlation between the time histories of the measured Neon soft X-ray pulse and the reflected shock phase of the computed current waveform which has been fitted to the measured current waveform. Results shows that the characteristic neon SXR measured at $3.17 \mathrm{~J}$ with a pulse duration of 249 ns starts typically after the radial inward shock phase and increases in magnitude few ns before the pinch phase. It tails unto the first anomalous resistance, and decays at the second anomalous resistance.
\end{abstract}

Keywords: Lee Model Code; SXR; reflective shock phase; plasma focus.

This is an Open Access article published by World Scientific Publishing Company. It is distributed under the terms of the Creative Commons Attribution 3.0 (CC-BY) License. Further distribution of this work is permitted, provided the original work is properly cited. 


\section{Introduction}

Dense plasma focus (DPF) is a pulsed electric gas discharge capable of producing neon SXR. Recently, it was found ${ }^{1}$ that plasma focus devices need to be classified into T1 and $\mathrm{T} 2$. The former having low static inductance $\mathrm{L}_{0}$ typically in the tens of $\mathrm{nH}$ while the latter typically have $\mathrm{L}_{0}$ of $100 \mathrm{nH}$ or more.

The INTI Plasma Focus machine is a T2 type machine having a high static inductance of $110 \mathrm{nH}^{2}{ }^{2}$ The Lee Model Code is extended to the current 6-phase to be able to fit the computed current waveform to the measured current waveform. It was found that the dynamics of the plasma focus (PF) computed by the 6-phase Lee Model Code is in agreement with the measured current waveform when the computed current waveform is properly fitted to the measured current waveform. This is to ensure that we get the time position of the SXR pulse relative to the plasma dynamics.

A valid question is worth asking, what is the correlation between the time history of the measured SXR pulse and the different phases (radial, reflected shock and pinch phase) of the computed current waveform? To answer this question involves the fitting procedures of the current waveform in the region of the radial phase accurately.

\section{The Lee Model Code}

The Lee Model Code couples the electrical circuit with plasma focus dynamics, thermodynamics and radiation, enabling realistic simulation of all gross focus properties. The code has been used extensively in several machines including UNU/ICTP PFF, ${ }^{3-7}$ $\mathrm{NX} 1{ }^{8} \mathrm{NX} 2,{ }^{5,8-9}$ and has been adopted for the Filippov-type DENA. ${ }^{10} \mathrm{~A}$ recent development is a template that shows the correlation of the neon SXR signal with the plasma focus dynamics ${ }^{2}$ and the inclusion of the anomalous resistance phase to simulate the Type 2 high inductance plasma focus machine. ${ }^{1}$ The Lee Model Code simulates the discharge evolution of the plasma dynamics shown in Fig. 1.

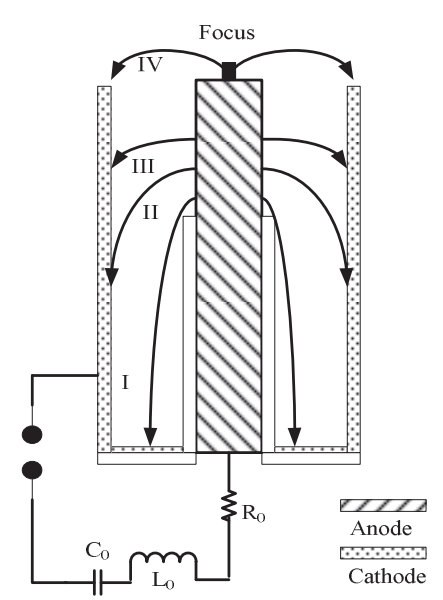

Fig. 1. The plasma focus with external capacitor $\mathrm{C}_{0}$, external inductance $\mathrm{L}_{0}$ switch and the focus tube. 
The discharge evolution consists of two main phases, the axial (I, II and III) and radial phase (IV). The axial phase starts with a breakdown phase (or inverse pinch). It refers to the electrical breakdown in a focus tube when a high voltage pulse is applied across the electrodes at an appropriate filling gas. An axial symmetric current sheath is formed at the end of this phase. This is followed by the axial acceleration phase (or axial rundown phase), whereby the current sheath is accelerated in the forward z-direction by the axial component of the Lorentz force towards the open end of the anode. The axial phase ends when the current sheath reaches the open end of the anode. The radial phase then begins. In the radial phase (or radial collapse phase) the current sheath implodes in a radially inward direction.

The evolution of the radial is divided into four sub-phases (see Fig. 2), namely, the radial inward shock phase (curves 1-2 and 1-3), radial reflected shock phase (curves 2-3 and 3-4), slow compression (curve 4-5) and the expanded column radial phase (curve 56). In the radial inward shock phase, the plasma slug is formed (point 1), then the magnetic piston radius $r_{p}$ and the shock front radius $r_{s}$ decreases continually until $r_{s}=0$ (point 3). This is followed by the reflected shock phase. The final phase, the pinch phase (curve 4-5) plays an important role in the plasma focus evolution because of its extremely high energy density. When neon is used, the neon pinch is a source of neon SXR. Finally, the plasma focus decays in an expanded column phase (5-6). ${ }^{11}$

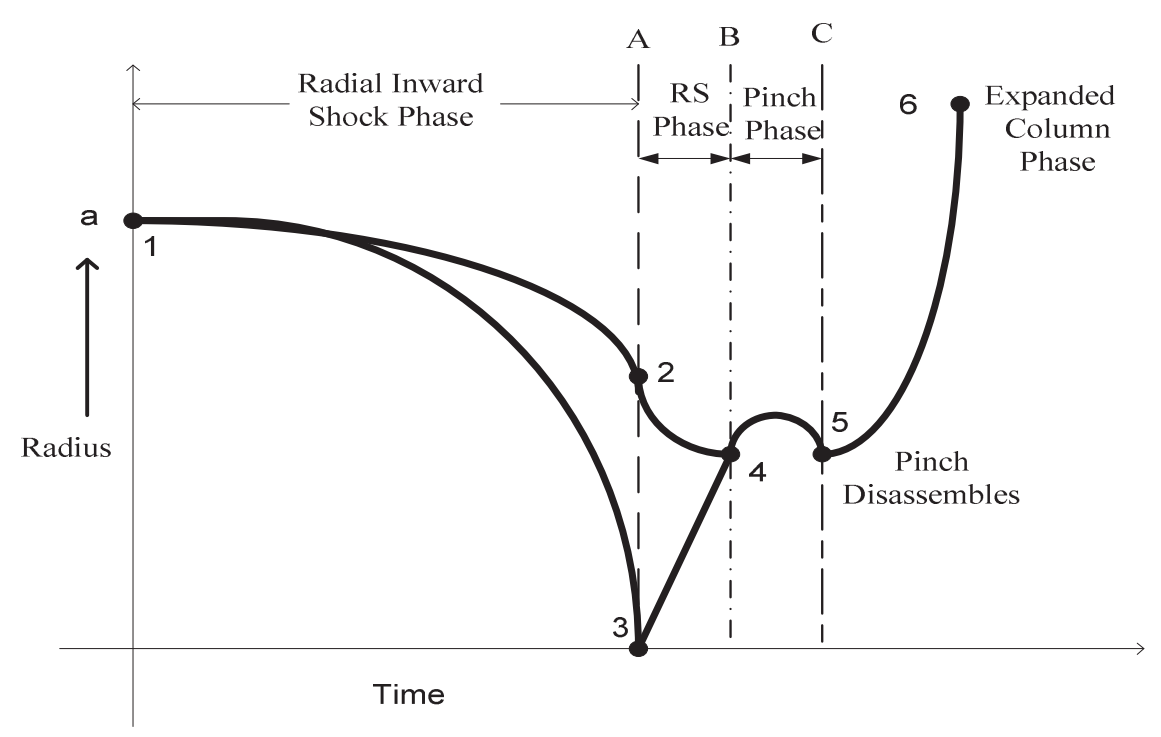

Fig. 2. The radial phase evolution where time markers A - start of the reflected shock phase, B - the end of the reflected shock phase and also the start of the pinch phase and $\mathrm{C}$ - the end of the pinch phase. 
The radial collapse phase plays the most important role in the plasma focus evolution due to its extremely high energy density and temperature. The focus pinch is a rich source of high energy particles and fusion products (when operated in deuterium) and also radiation such as soft $\mathrm{x}$ - rays. We note that the transition from slow compression (pinch phase) to the expanded column is observed in the laboratory to be in an extremely short time with plasma/current disruptions which resulted in localized regions of high densities and temperatures. These localized regions are not modelled in the code, which computes only average uniform density and temperature.

However, since the code incorporates four model parameters $f_{m}$ and $f_{c}$ (mass and current factors for the axial phase) and $f_{m r}$ and $f_{c r}$ (mass-swept-up and current factor in the radial phase) by fitting the computed to the measured total current waveform, the model incorporates the energy and mass balances equivalent to other processes which are not modelled. Therefore, the computed gross features such as speeds and trajectories and integrated neon SXR yields have been extensively tested in numerical experiments for several machines and found to be comparable with measured values.

In the Lee Model Code, ${ }^{12}$ neon line radiation $\mathrm{Q}_{\mathrm{L}}$ is calculated as follows:

$$
\frac{d Q_{L}}{d t}=-4.6 \times 10^{-31} n_{i}^{2} Z Z_{n}^{4}\left(\pi r_{p}{ }^{2}\right) Z_{f} / T
$$

where, for the temperatures of interest in our experiments, we take the neon SXR yield Y SXR $=Q_{L}$, where $Z_{n}$ is the atomic number. Since in our code, is obtained by integrating over the pinch duration, the neon SXR energy generated within the plasma pinch depends on the following properties: number density, effective charge number Z, pinch radius, pinch length, and temperature $\mathrm{T}$ and pinch duration.

This generated energy is affected by plasma self-absorption which depends on density and temperature; the reduced quantity of energy is then emitted as the neon SXR yield. These effects are compensated in the model by computing the volumetric plasma selfabsorption factor "A" derived from the photonic excitation number $\mathrm{M}$ which is a function of, $\mathrm{Z}$ and $\mathrm{T}$. However in our range of operation, the numerical experiments show that the plasma self-absorption is not significant. It was pointed out by Liu, ${ }^{4}$ that the temperature around $300 \mathrm{eV}$ is optimum for neon SXR production. Bing's subsequent work, ${ }^{13}$ and our experience through numerical experiments suggest that around $2 \times 10^{6} \mathrm{~K}$ (below $200 \mathrm{eV}$ ) or even a little lower could be better. Hence, for neon SXR scaling, there is a range of temperature (T windows) suitable for efficient emission of neon SXR. For SXR it is important that the pinch temperature is within the SXR emission window. If it is within the $\mathrm{T}$ window, the computed $\mathrm{Q}_{\mathrm{L}}$ is mainly neon SXR. If $\mathrm{T}$ is too high, the neon plasma is fully ionized, hence very little of the $\mathrm{Q}_{\mathrm{L}}$ is $\mathrm{SXR}$. If $\mathrm{T}$ is too low, the neon plasma is not hot enough to ionize to the He-like $\mathrm{H}$-like levels, and the $\mathrm{Q}_{\mathrm{L}}$ is mainly $\mathrm{UV}$ and visible lines. 


\section{Experimental Set Up and Methodology}

The electrical set up of the apparatus and diagnosis systems are shown in Fig. 3. The experiment was carried out at a constant charging voltage of $12 \mathrm{kV}$ and using neon as the working gas. The neon SXR pulses (from 2 detector channels of diode X-ray spectrometer DXS) and the measured rate of change of current dI/dt and the tube voltage are recorded together on a 4-channel Digital Storage Oscilloscope DSO at a sample rate of $1 \mathrm{GSa} / \mathrm{s}$ at a frequency response of $200 \mathrm{MHz}$. The voltage signal is obtained using a resistive divider with a time response in the region $15 \mathrm{~ns} .{ }^{14}$ The $\mathrm{dI} / \mathrm{dt}$ signal is from a 7- turn Rogowski coil wrapped around one of the 16 conductors returning the current from the focus tube to the capacitor earth. ${ }^{15}$ The absorption filters method based on foil absorbers and silicon PIN diode detectors is used in the SXR spectrometer (see Fig. 4). The two neon SXR detectors of the DXS are used together as a differentially filtered pair, Ch1 and Ch2 of DXS, to measure the characteristic He-like and H-like neon line SXR by the method of subtraction. ${ }^{1}$ Each detector consists of a reverse-biased windowless BXP65 PIN photodiode with a wide spectral range. In designing the required filter the emission wavelength with expected line intensities are suitably weighted and factored in to obtain the average sensitivity factor for the relatively narrow spectral window of 900-1550 eV. The first detector is covered with $13 \mu$ aluminum (SXR Ch1) while the second detector is covered with $125 \mu$ mylar film $+3 \mu$ aluminum (SXR Ch2).

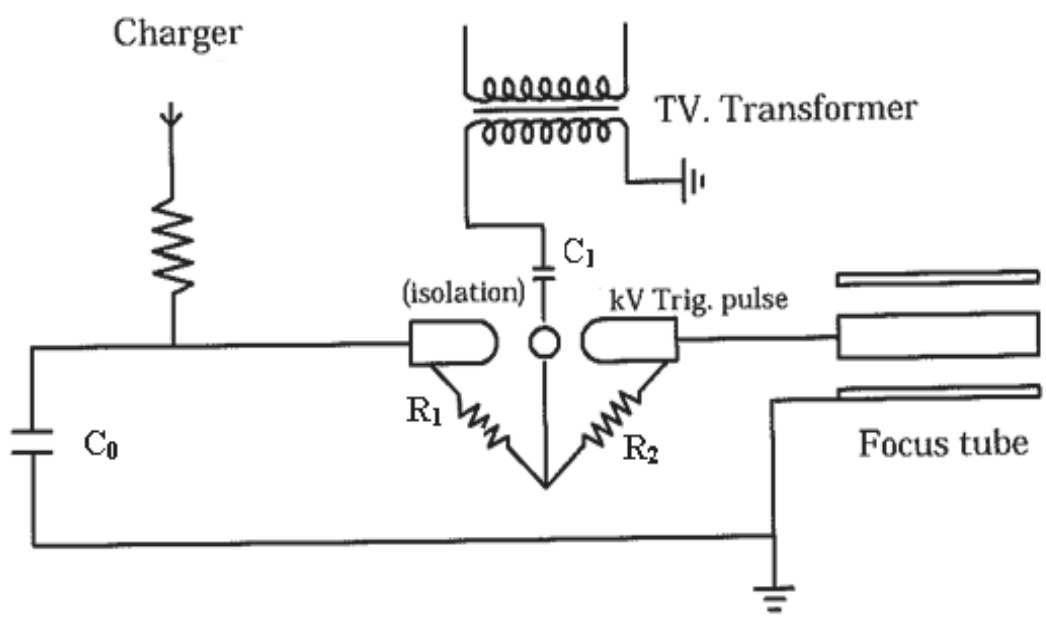

Fig. 3. Circuit for the swinging-spark gap and focus tube. 


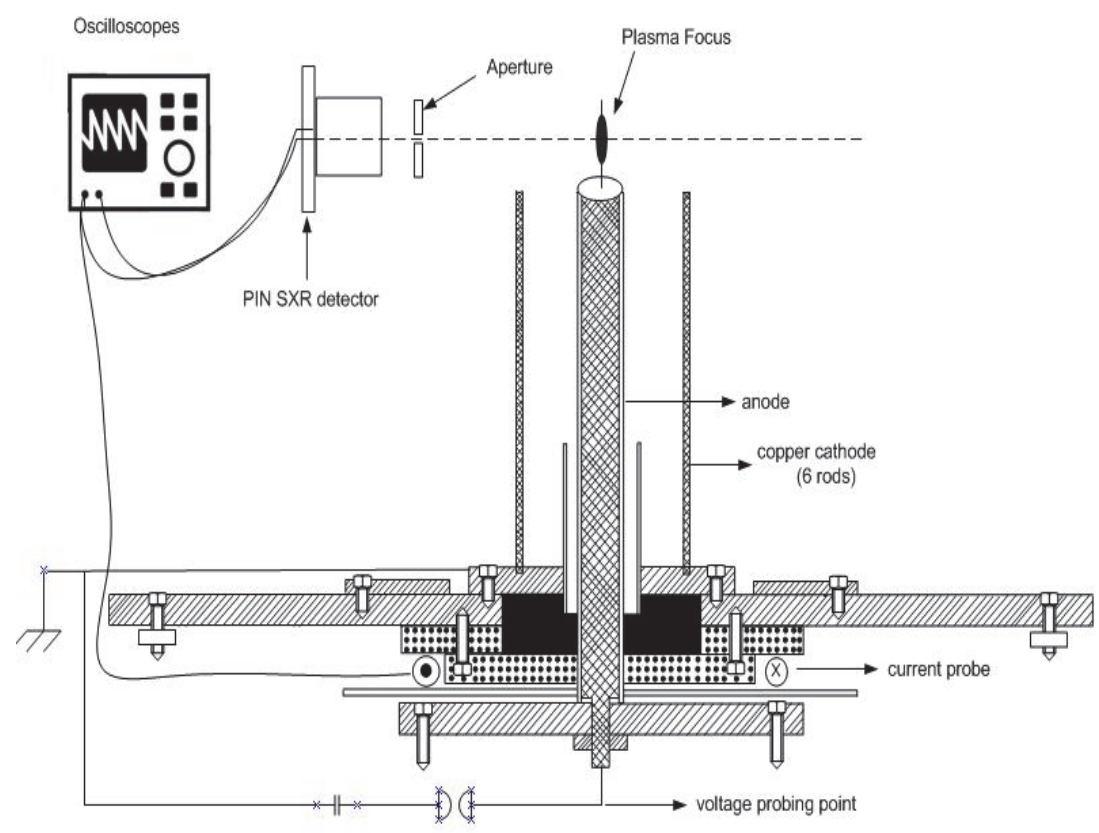

Figure 4: SXR measurement in a DPF.

Figure 5 shows the sensitivity of each channel for this pair as a function of photon energy in electron volts. At the scale in Fig. 5, the two sensitivity curves are identical and appear as one overlapped curve from 0 to $20,000 \mathrm{eV}$, except for the sharp spike on the left side of the curve with a photonic energy range of 900-1550 eV. This spike (transmission window) belongs to Ch 1 only. The filter pair, for Ch1 and Ch2, is designed to give a difference window in the spectral region of the characteristic He-like and H-like SXR emission from neon 900-1550 eV. ${ }^{16}$ Subtracting the signal of Ch2 from that of Ch1 thus allows the amount of characteristic neon SXR falling on the detectors to be computed. For example, if, for a shot, the difference signal between the two channel is zero, this means that no emission in the $900-1550 \mathrm{eV}$ is detected. If there is a difference, it can only come from the $900-1550 \mathrm{eV}$ window, and the difference signal is a measure of the characteristic neon SXR detected. On the other hand, if pulses are detected by the channels have no difference signal; each channel is detecting a signal with photon energy above $1550 \mathrm{eV}$. This signal is an SXR harder than the characteristic neon SXR emission.

Therefore, capturing the SXR pulse on a DSO, the amount of characteristic neon line SXR falling on the detector may be measured, and by assuming a point source radiating isotropically, the source yield can be estimated by space integrating over $4 \pi$ and time integrating over the duration of the pulse. Both detectors have been normalized to one 


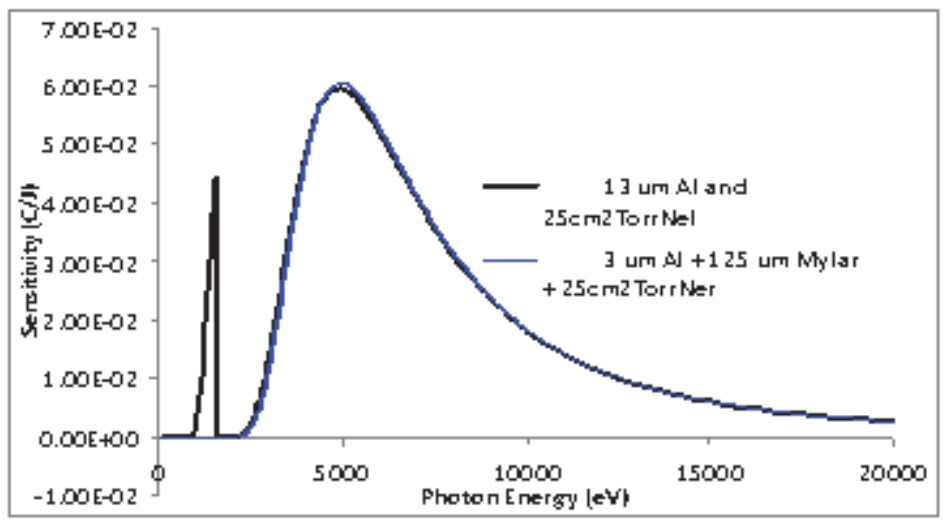

Fig. 5. The sensitivity of the two $\mathrm{Ch} 1$ and $\mathrm{Ch} 2$ are designed to have identical sensitivity curves for the region from around $1550 \mathrm{eV}$ upward to $20000 \mathrm{eV}$ and beyond. The only difference in the two curves is that $\mathrm{Ch} 1 \mathrm{has}$ an additional region of sensitivity from 900 to $1550 \mathrm{eV}$ (the spike like feature between 0 and $2500 \mathrm{eV}$ in the figure belongs to Ch1 only).In the other spectral (or photon energy) regions, there are two curves on top of each other, so the two curves are seen as one.

another and are positioned side by side with the same distance to the focus position where the focus pinch emits the radiation to be detected. In this way, the experimentally measured current, tube voltage, and characteristic neon SXR yield are time correlated and ready for comparison with the time variations of computed current and trajectories.

\section{The Results}

To start the numerical experiments, we select a discharge current trace of the INTI Plasma Focus machine taken with a Rogowski coil. The following bank tube and operation parameters are used:

1) Bank: static inductance $\mathrm{L}_{0}=119 \mathrm{nH}, \mathrm{C}_{0}=30 \mu \mathrm{F}$, and stray resistance $\mathrm{r}_{0}=12.5 \mathrm{~m} \Omega$

2) Tube: cathode radius $\mathrm{b}=3.2 \mathrm{~cm}$, anode radius $\mathrm{a}=0.95 \mathrm{~cm}$ and anode length $\mathrm{z}_{0}=16 \mathrm{~cm}$.

3) Operation: voltage $\mathrm{V}_{0}=12 \mathrm{kV}$ and pressure $=3$ Torr neon

The computed total discharge current waveform is fitted to the measured current trace. Fitting involves sequential variations in all the parameters such as $f_{m}, f_{c}, f_{m r}$ and $f_{c r}$ for the axial and radial phases. These parameters are sequentially varied until a visually acceptable fit between the measured and computed current trace is obtained. The following fitted model parameters are obtained: $f_{m}=0.0252, f_{c}=0.7, \mathrm{f}_{m r}=0.2$ and $f_{c r}=0.8$. The computed and measured current trace is shown in Fig. 6. It also shows the outputs of the two channels of SXR and the voltage waveform. The four measured waveforms are displayed on the same 4-channel DSO. 


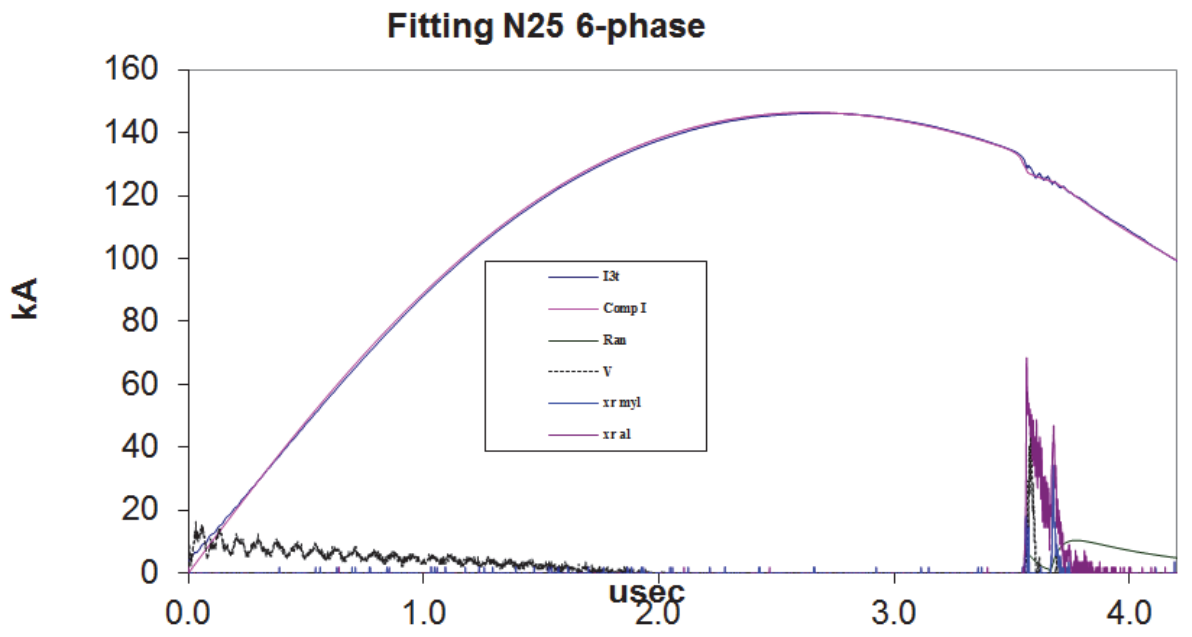

Fig. 6. Correlating currents and SXR channels. The computed current (pink) is fitted so well to the measured current (blue) that the two traces overlap, appearing as one trace (the top trace). SXR Ch 1 (purple) and Ch 2 (blue) are shown. In addition, V (black) and anomalous resistance (green) are included as well.

Figure 7 shows that the computed current waveform is fitted very well to the measured current waveform, the two waveforms lying on top of each other appearing as one up to the point $\mathrm{t}=4.158 \mu \mathrm{s}$.

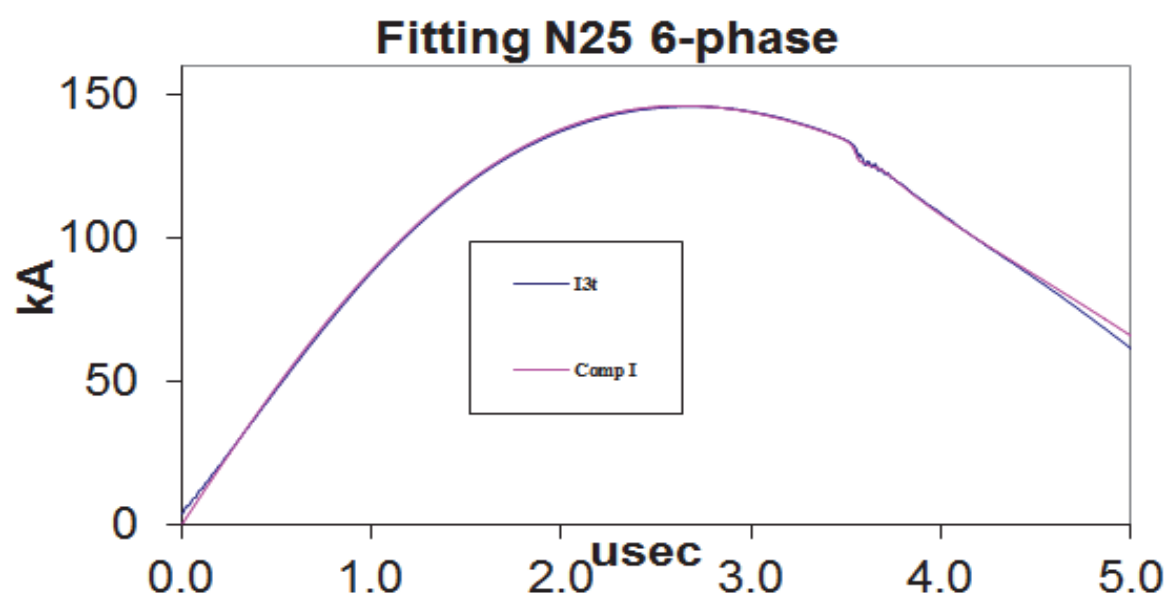

Fig. 7. Well fitted measured (blue) and computed (pink) current trace. 
Figure 8 is an expansion of Figs. 6 and 7 for the region $3.3 \mu$ s to $3.8 \mu \mathrm{s}$. It shows the outputs of the two SXR channels. From this Fig. 8, we see that the SXR pulse is characteristic neon SXR (since there is a difference pulse). The characteristic neon SXR starts $2 \mathrm{~ns}$ (at $3.551 \mu \mathrm{s}$ ) after the radial inward shock phase which is also the beginning of the radial shock phase. This confirms the findings of Lee et al, ${ }^{1}$ that the characteristic neon SXR starts before the start of the pinch. In this particular shot, the neon SXR starts $7 \mathrm{~ns}$ before the start of the pinch (at $3.558 \mu \mathrm{s}$ ), increases and reached the maximum amplitude in the pinch phase (at $3.562 \mu \mathrm{s}$ ). The two SXR channels coincide at a shorter time of $7 \mathrm{~ns}$ ( $3.551 \mu \mathrm{s}$ to $3.558 \mu \mathrm{s})$. Afterwards, the neon characteristic SXR decreases in amplitude in the first anomalous resistance AR1 (at $3.658 \mu \mathrm{s}$ ) with duration of $107 \mathrm{~ns}$. After the first AR1, the characteristic neon SXR increases at lower amplitude in the second anomalous resistance AR2 with duration of $142 \mathrm{~ns}$. Finally, the SXR pulse decays at $3.8 \mu \mathrm{s}$. Also shown in Fig. 8 are time markers (vertical line) indicating from the computed results the start of the radial phase (solid vertical line), the end of the radial inward shock phase (red dashed line) which is also the beginning of the radial shock phase, the start and end of the pinch phase (blue dashed line). This characteristic He-like and H-like neon SXR pulse for this shot is integrated and found to have a radiation yield of $3.17 \mathrm{~J}$.

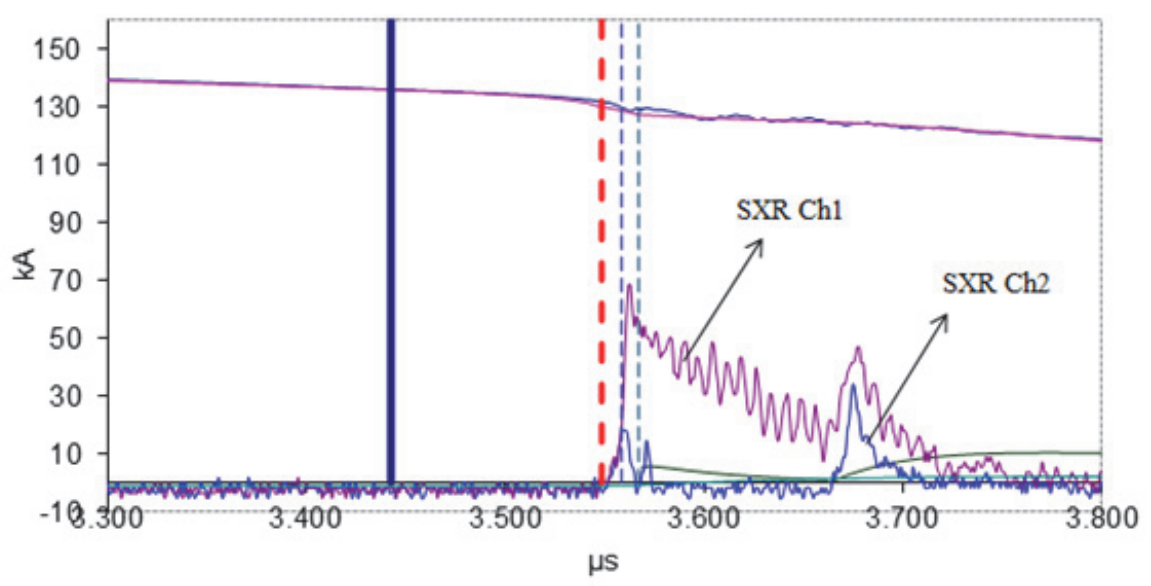

Fig. 8. This is an expansion of Figs. 6 and 7 from 3.3 to $3.8 \mu$ s. The top trace running across the figure is the overlapped lines of the computed and measured currents. The SXR Ch1 (purple) and Ch2 (blue) overlaps at the beginning.

Analysis of a number of shots between 2.5 and 3 Torr at $12 \mathrm{kV}$ neon shows that the correlation of Fig. 7 is typical of most of the shots. The characteristic neon SXR line yields of these typical shots lies in the range 1 to $4 \mathrm{~J}$. 


\section{Conclusions}

The characteristic He-like and H-like neon line SXR pulse found out to typically start in the radial shock phase or between the end of the radial inward shock phase and the start of the pinch phase. Finally, the pulse increases in amplitude in the pinch phase and decreases in the anomalous resistance phase.

These results have suggestions in improving the model code for the calculation of characteristic He-like and H-like SXR yield.

\section{Acknowledgments}

The authors would like to give gratitude's to the Office of Research and Development and the Centre for Plasma Research of the INTI International University for all the moral and financial supports extended to the author and the organizer of the ICPSA 2013 for the acceptance of the abstract submitted.

\section{References}

1. S. Lee, S. H. Saw, A. E. Abdou, and H. Torreblanca, Characterizing plasma focus devices-role of the static inductance-instability phase fitted by anomalous resistances.,J Fusion Energy 30, 277-282 (2011).

2. S. Lee, S. H. Saw, P. Lee, A. Tabelaher, A. E. Abdou, P. L. Chong, F. A. Roy Jr., A. Singh, D. Wong and K. Devi, Correlation of measured soft X-ray pulses with modeled dynamics of the plasma focus, IEEE Trans on Plasma Sci 39,.3196-3202 (2011).

3. S. Lee, T. Y. Tou, S. P. Moo, M. A. Eissa, A. V. Gholap, K. H. Kwek, H. Mulyodrono A. J. Smith, S. Suyadi, W. Usada and M. Zakaullah, A simple facility for the teaching of plasma dynamics and plasma nuclear fusion. Amer. J. Phys., 56, 62-68 (1988).

4. M. Liu, Soft X-rays from compact plasma focus, Ph.D dissertation, NIE, Nanyang Technological University, Singapore (2006).

5. S. Lee, and A. Serban, Dimensions and lifetime of the plasma focus pinch, IEEE Trans. Plasma Sci., 24, 1101-1105 (1996).

6. S. Lee, In Twelve Years of UNU/ICTP PFF- A Review, Trieste,Italy: Abdus Salam ICTP, IC/98/231, ICTP ,(1998), pp.5-34.

7. S. Lee, In Twelve Years of UNU/ICTP PFF- A Review, Trieste,Italy: Abdus Salam ICTP, IC/98/231, ICTP ,(1998), pp.5-34.

8. S. Lee,P. Lee, G. Zhang, A. Patran, X. Feng, V. A. Gribkov, M. Liu, A. Serban and T. Wong, High rep rate high performance plasma focus as a powerful radiation source, IEEE Trans.Plasma Sci., 26, 1119-1126 (1998).

9. D. Wong, P. Lee, T. Zhang, A. Patran, T. L. Tan, R. S. Rawat and S. Lee, An improved radiative plasma focus model calibrated for neon-filled NX2 using tapered anode. Plasma Sources Sci. Technol. 16, 116-123 (2007).

10. V. Sialpoush, M. A. Tafreshi, S. Sobhanian and S. Khorram, Adaptation of Sing Lee's model to the Filippov type plasma focus geometry.Plasma Phys.Control.Fusion 47, 1065-1075 (2005).

11. F. A. Roy Jr., P. L. Chong, S. H. Saw and S. Lee, Generation of Soft X-Ray from Plasma Focus Machine. International Conference on Recent and Emerging Advanced Technologies in Engineering (2009). 
12. S. Lee, Radiative Dense Plasma Focus Computation Package: RADF.[Online]. Available: http://www.intima.edu.my/school/fas/UFLF/File1RADPF.htm,//www.plasmafocus.net/IPFS/ modelpackage/File1RADPF.htm

13. S. Bing, Plasma dynamics and X-ray emission of the plasma focus, Ph D dissertation, NIE, Nanyang Technological Univ., Singapore (2000).

14. S. Lee, T. Y. Tou, , S. P. Moo, M. A. Eissa, A. V. Ghol, K. H. Kwek, S. Mulyodrono, A. J. Smith, W. Usada, and M. Zakaullah, A simple facility for teaching of plasma dynamics and plasma nuclear fusion, Amer. J. Phys. 56, 62-68 (1988).

15. S. H. Saw, S. Lee, F. Roy, P.L. Chong, V. Vengadeshwaran, A. S. M. Sidik, Y.W. Leong and A. Singh, In situ determination of static inductance and Resistance of a plasma focus capacitor bank, Rev. Sci. Instrum. 81, 053505 (2010).

16. S. H. Saw, P. C. K. Lee, T. Ong, S. N. Mohamad, F. D. Ismail, N. D. Nawi, K. Devi, R. M. Sabri, A. H. Baijan, J. Ali, and S. Lee, Magnetic Probe Measurements in INTI Plasma Focus to determine dependence of axial speed with pressure in Neon, J. Fusion Energ 31, 411-417 (2012). 